\title{
CIRURGIA VIDEOLAPAROSCÓPICA NAS DOENÇAS BILIOPANCREÁTICAS
}

\section{LAPAROSCOPIC SURGERY IN PANCREATOBILIARY DISEASES}

\author{
Luiz Rohde, TCBC-RS ${ }^{1}$ \\ Daniel Melecchi de Oliveira Freitas ${ }^{2}$ \\ Alessandro Bersch Osvaldt, ACBC-RS ${ }^{3}$ \\ Priscila Viero ${ }^{2}$ \\ Vivian Pierri Bersch, RCBC-RS ${ }^{3}$
}

\begin{abstract}
RESUMO: Com a experiência adquirida na cirurgia da vesícula biliar, a videocirurgia está sendo indicada cada vez mais em outras doenças. Decorridos 15 anos da primeira cirurgia, os autores avaliam os resultados e analisam as perspectivas deste procedimento nas doenças biliopancreáticas. Seguem a proposta que divide este procedimento em procedimentos de rotina, avaliação e desenvolvimento. Nas doenças da vesícula biliar, a colecistectomia laparoscópica é considerada padrão ouro, levando vantagem em todos os itens sobre a laparotômica, excluídas as lesões da via biliar e o vazamento biliar pelo coto cístico. Enfatizam situações especiais: da colecistite aguda, da colecistectomia durante a gravidez, da vesícula em porcelana e do câncer da vesícula. Comentam as perspectivas da videocirurgia nas complicações da colecistectomia laparoscópica. No tratamento da coledocolitíase, consideram o procedimento como em avaliação pela falta de ensaios clínicos prospectivos randomizados com grupo controle comparáveis e acompanhados por prazo de tempo maior. Nas doenças do pâncreas, a videocirurgia é um procedimento em desenvolvimento, com exceção da pancreatite aguda biliar não complicada, que se beneficia com a colecistectomia laparoscópica. O mesmo ocorre com as cirurgias de derivação para desobstrução da via biliar. Embora factíveis dentro dos princípios da cirurgia convencional, faltam estudos comparativos com outras técnicas existentes analisando eficácia e efetividade.
\end{abstract}

Descritores: Colecistectomia videolaparosocópica; Videocirurgia; Videocoledocolitotomia; Vias biliares; Pâncreas.

\section{INTRODUÇÃO}

A primeira colecistectomia laparoscópica foi realizada em 1985 por Mühe, na Alemanha, sem videocirurgia ${ }^{1}$. A partir de 1987, com Mouret \& Dubois², o método passou a ser mais divulgado, substituindo gradativamente a colecistectomia por laparotomia. Nos dias de hoje, a colecistecto- mia videolaparoscópica é considerada o padrão ouro no tratamento da colelitíase ${ }^{3}$.

Com a experiência adquirida na cirurgia da vesícula biliar, a videocirurgia está sendo indicada cada vez mais em outras doenças. Decorridos quase 15 anos da primeira cirurgia, é objetivo deste trabalho uma avaliação dos resultados obtidos e uma análise das perspectivas nas doenças biliopancreáticas.

1. Professor Titular do Departamento de Cirurgia, Faculdade de Medicina da UFRGS.

2. Bolsistas de Iniciação Científica.

3. Alunos do Programa de Pós-Graduação em Cirurgia.

Recebido em 10/12/99

Aceito para publicação em 22/5/2000

Trabalho realizado no Departamento de Cirurgia da Faculdade de Medicina da Universidade Federal do Rio Grande do Sul (UFRGS) e no Serviço de Cirurgia Geral, Grupo de Vias Biliares e Pâncreas do Hospital de Clínicas de Porto Alegre — Rio Grande do Sul. 


\section{EM DOENÇAS DA VESÍCULA BILIAR}

Inúmeros trabalhos demonstram que a colecistectomia videolaparoscópica (CVL) supera em resultados a colecistectomia por laparotomia (CL). O tempo de internação é menor, o paciente sente menos dor, a recuperação é mais rápida, com retorno ao trabalho mais precoce, os custos praticamente se equivalem e as complicações são menores $^{4-6}$. Só a lesão da via biliar e o coleperitônio decorrente de vazamento biliar pelo coto cístico são mais freqüentes na $\mathrm{CVL}^{7,8}$. A lesão de via biliar ocorre numa frequiência que varia de $0 \%$ a $0,4 \%$ na $\mathrm{CL}^{9,10}$ e numa freqüência que varia entre $0 \%$ a $1,8 \%$ na $\mathrm{CVL}^{6,11,12}$. Em termos médios, naquela ocorre em $0,1 \%$ e nesta em $0,6 \%$. Ainda é, portanto, seis vezes mais freqüente na CVL e ocorre com mais freqüência na via biliar proximal, sendo mais grave ${ }^{13}, 14$. A incidência de vazamento biliar referida na literatura na CL varia consideravelmente. Alguns trabalhos publicados não referem esta complicação ${ }^{15,16}$, outros mostram uma incidência que varia de $0,3 \%$ a $0,5 \% .^{9,10}$. Na CVL, ocorre com mais frequiência. Wolfe ${ }^{11}$ refere esta complicação em 1,04\% dos casos e Peck $^{17}$ em 0,6\%. Em 1965 CVL revisadas ${ }^{18}$, ocorreram 10 lesões, numa percentagem de $0,5 \%$ e 12 vazamentos da via biliar pelo coto cístico numa percentagem de $0,6 \%$.

A não utilização do eletrocautério próximo ao pedículo para evitar lesões térmicas, o uso de ótica de $30^{\circ}$ para visualização lateral do pedículo, a colangiografia intra-operatória que pode não prevenir, mas auxilia na identificação precoce de lesões, são recomendações para diminuir estas intercorrências.

A discussão sobre a realização da colangiografia intraoperatória de rotina ou seletiva permanece a mesma da era anterior à CVL.

Com o aperfeiçoamento do instrumental utilizado e o aumento da experiência neste tipo de procedimento, espera-se que estas complicações citadas diminuam, reforçando ainda mais o papel da CVL no tratamento das doenças da vesícula biliar.

Cerca de $85 \%$ das colecistites agudas são resolvidas pela CVL. São convertidas para a cirurgia aberta aquelas em que o processo inflamatório bloqueia de tal forma a região, que fica impossível identificar as estruturas, embora em situações especiais possa ser realizada a colecistectomia parcial ${ }^{19}$. A síndrome de Mirizzi quase sempre exige a conversão, especialmente a do tipo III.

A colelitíase sintomática é a segunda causa mais prevalente de abdômen agudo na gestação. Nas pacientes em que o tratamento é conservador, as reinternações são 50\% superiores e em $16 \%$ das pacientes ocorre aborto espontâneo ou trabalho de parto prematuro ${ }^{20}$. O segundo trimestre da gravidez é o ideal para a cirurgia. A chance de aborto é quase o dobro quando a cirurgia é realizada no $1^{\circ}$ trimestre. No terceiro trimestre, o risco é o de trabalho de parto prematuro. O tamanho do útero gravídico prejudica a colocação de trocartes. É uma boa prática introduzir primeiro trocarter supraumbilical pela técnica aberta (Hasson). É recomendável manter o pneumoperitônio com baixa pressão $(<12 \mathrm{mmHg})$, pois acima de $15 \mathrm{mmHg}$ pode provocar hipercarbia e acidose fetal. O útero deve ser deslocado le- vemente para a esquerda para evitar compressão da veia cava inferior que dificulta o retorno venoso com conseqüente diminuição do débito cardíaco e do fluxo sangüíneo placentário. A exposição do feto à radiação deve ser evitada na eventualidade da colangiografia intraoperatória, especialmente no primeiro trimestre da gravidez. A CVL na mulher grávida deve ser considerada um procedimento em avaliação. Não existem trabalhos científicos em grande série e desconhece-se o efeito do pneumoperitônio no feto a longo prazo. Trabalhos experimentais demonstram a ocorrência de uma acidose fetal que sugere um potencial efeito adverso sobre a criança ${ }^{21,22}$. Outros trabalhos, no entanto, referem a CVL na vigência da gravidez como um procedimento seguro para a mãe e o feto ${ }^{23}$.

Em aberto permanece também a conduta na vesícula em porcelana. Muitas delas não oferecem um plano que permita a colocação de dois clipes e secção do conduto cístico, especialmente porque em cerca de $20 \%$ dos casos já se trata de um adenocarcinoma de vesícula ${ }^{24}$. Quando existem indícios que se possa tratar de câncer de vesícula, a CVL é contra-indicada pela possibilidade de implantação tumoral $^{25,26}$. Da mesma forma, quando no intraoperatório a ecografia laparoscópica sugere câncer de vesícula ${ }^{27}$. Quando o diagnóstico é feito no pós-operatório, é recomendável, além da cirurgia indicada na dependência do estadiamento do tumor, a ressecção em torno do trajeto dos trocartes colocados durante a videocirurgia ${ }^{28,29}$.

Embora os critérios de indicação da cirurgia na colelitíase permaneçam os mesmos da era anterior à videocirurgia, com a progressiva melhora dos resultados com a CVL em termos de morbidez, mortalidade e tempo de recuperação, a indicação de cirurgia na colelitíase assintomática começa a ser discutida. Não existem, por ora, argumentos suficientes para sustentar esta conduta.

\section{NAS COMPLICAÇÕES DA VIDEOCOLECISTECTOMIA}

Complicações da CVL que no início da experiência eram tratados com laparotomia podem ser resolvidos com nova videocirurgia. Vazamentos da via biliar pelo cístico podem ser tratados por papiloesfincterotomia endoscópica associada ou não a uma nova clipagem do coto cístico ${ }^{7}$. Como esta complicação não é tão infreqüente, é de se considerar a ligadura do cístico com fio, como é feito na cirurgia aberta. Além de fechar o ducto com mais segurança, serviria especialmente em hospitais-escola como treinamento das habilidades necessárias para trabalhar sobre o colédoco e diminuiria o custo da cirurgia ${ }^{30}$.

Da mesma maneira, nos casos em que ocorre clipagem indevida da via biliar principal, diagnosticada no pósoperatório pela colangiopancreatografia endoscópica retrógrada (CPER), a simples retirada do clipe por nova videocirurgia poderá ser o tratamento indicado (embora a médio prazo possa ocorrer estenose cicatricial, sobretudo pelo quase sempre diminuto diâmetro da via biliar nestes casos).

Na experiência dos autores ${ }^{18}$ em 10 lesões da via biliar, ocorreu pequena secção da via biliar principal em dois ca- 
sos durante o descolamento do infundíbulo vesicular do colédoco não diagnosticados durante $\mathrm{o}$ ato operatório pela falta de vazamento biliar (não presente por causa da pressão exercida pelo pneumoperitônio). Diagnosticada esta complicação pela CPER, também pode ser tratada por nova videocirurgia com colocação de dreno de Kehr e/ou sutura.

\section{NA COLEDOCOLITÍASE}

Seis anos depois da primeira CVL, apareceram os trabalhos relacionados com a extração de cálculos do colédoco pela videocirurgia ${ }^{31}$ com eficácia (grau com que um procedimento é capaz de produzir o efeito benéfico desejado) e efetividade (considera recursos materiais, humanos e tem conotação econômica) praticamente superponíveis ao da cirurgia aberta ${ }^{32}$. No nosso país, o primeiro trabalho data de $1994^{33}$. Embora factível e seguindo os princípios da cirurgia aberta, o procedimento ressente-se de um ensaio clínico prospectivo randomizado com grupos controle comparáveis e de um acompanhamento a longo prazo dos pacientes operados. Os resultados de uma intervenção melhoram à medida que a experiência aumenta. Como a coledocolitíase é bem menos freqüente que a colelitíase (em média, no passado, na proporção de 12:100 e nos dias de hoje na de 8:100), uma experiência maior só será adquirida em centros de referência. O cirurgião que realiza dois CVL por semana, fará oito por mês e $96 \mathrm{em}$ um ano. Terá a provável experiência com o tratamento de sete coledocolitíases. Certamente, este é um fator limitante para a difusão do procedimento. Acrescente-se ainda a possibilidade do tratamento endoscópico possível na maioria dos casos com resultados similares aos tratamentos cirúrgicos por vídeo ou por cirurgia aberta. Assim, o manejo dos cálculos da via biliar extra-hepática na era da laparoscopia é controver$\mathrm{so}^{34}$. A perspectiva por ora é de que a videocoledocolitomia fique reservada ainda aos centros especializados.

Embora um trabalho recente ${ }^{35}$ considere a exploração laparoscópica do ducto biliar comum como padrãoouro, ainda falta experiência mundial suficiente para considerá-la como tal. É procedimento que ainda está em avaliação. Pelo fato de a videocoledocolitotomia seguir os princípios da cirurgia aberta e num só ato resolver o problema do doente com o aumento da experiência, ela tem condições para se firmar como procedimento de escolha. A cirurgia aberta permanece como indicação quando falta experiência com o procedimento e/ou instrumental e/ou quando há falha do tratamento endoscópico ou contraindicações do pneumoperitônio. Tem indicação ainda na dependência do tamanho, do número e da localização dos cálculos, do diâmetro da via biliar, da necessidade de cirurgia de derivação, da presença de outras afecções não tratáveis por videocirurgia e ainda na litíase residual quando falham o tratamento endoscópico e outras formas de tratamento.

Quanto ao uso da ecografia laparoscópica para o diagnóstico da coledocolitíase, os resultados são por enquanto superponíveis aos achados da colangiografia intra-operatória ${ }^{27}$.

\section{EM DOENÇAS DO PÂNCREAS}

Na pancreatite aguda biliar, a CVL com colangiografia intra-operatória está indicada assim que haja evidência de remissão do processo inflamatório. Na presença de critérios clínicos e laboratoriais de coledocolitíase, está indicada a CPER no pré-operatório quando não existem condições para exploração laparoscópica da via biliar principal.

Existem relatos do uso da cirurgia laparoscópica na pancreatite aguda complicada com necrose e abscessos ${ }^{36}$. São experiências isoladas que precisam ser confirmadas através de trabalhos no futuro. Elas têm como boa perspectiva a possibilidade da permanência dos trocartes, permitindo com facilidade uma segunda visão.

O tratamento do pseudocisto de pâncreas por laparoscopia, especialmente o de localização retrogástrica, segue a mesma estratégia da cirurgia aberta descrita por Jurasz. Ainda são poucas as referências, mas as perspectivas são animadoras ${ }^{37}$.

A laparoscopia vem sendo utilizada cada vez mais para o estadiamento das neoplasias malignas do pâncreas, antecedendo a realização do ato cirúrgico ${ }^{38}$. Os exames de imagem subavaliam a doença. Não é incomum encontrarem-se implantes peritoniais e pequenas metástases hepáticas não vistas pela tomografia computadorizada, o que poderá mudar a conduta no tratamento do doente. Da mesma maneira, a ecografia transoperatória por laparoscopia melhora a sensibilidade na detecção de metástases hepáticas e irressecabilidade por invasão de estruturas vasculares $^{27}$. A citologia positiva para células neoplásicas de líquido peritonial (às vezes em pequena quantidade e só diagnosticada por laparoscopia) tem implicação no estadiamento e no prognóstico ${ }^{39}$.

Série de casos com cinco a 12 pacientes portadores de tumores benignos das ilhotas do pâncreas referem benefício com tratamento laparoscópico por enucleação ou por pancreatectomia distal ${ }^{40}$. As vantagens estão relacionadas com a incisão e menor morbidade e a desvantagem na impossibilidade da palpação da glândula e a dificuldade de explorar o pâncreas em sua totalidade. Existem também relatos de ressecção de cistoadenomas com sucesso ${ }^{41}$.

Quanto à duodenopancreatectomia laparoscópica, embora factível não demonstra ainda vantagens sobre a cirurgia aberta ${ }^{42}$. A primeira foi realizada em 1992. Desde então têm sido relatados casos esparsos, mas não existe experiência suficiente.

A laparoscopia no tratamento da pancreatite aguda complicada, dos pseudocistos, dos tumores benignos e malignos do pâncreas é procedimento em desenvolvimento.

\section{CIRURGIAS DE DERIVAÇÃO}

Coledocoduodeno, colecisto e coledocojejunoanastomoses por laparoscopia têm sido realizados para desobstrução da via biliar ${ }^{43}$. Especialmente as duas primeiras são mais facilmente realizáveis. Seguem os princípios da cirurgia aberta e permitem anastomose livre de tensão e aposição de mucosa com mucosa, com as vantagens da cirurgia minimamente invasiva. No entanto, as publicações existentes refe- 
rem mais relatos de casos, ainda que em número pequeno. A literatura ressente-se da falta de trabalhos comparativos so- bre a derivação biliar laparoscópica com a colocação de stents por endoscopia e por punção percutânea trans-hepática.

\begin{abstract}
With the acquired experience with laparoscopic cholecystectomy, videosurgery has been used more and more for treatment of other diseases. Since the first surgery, nearly 15 years ago, the authors evaluate the outcome and the perspectives of this procedure in pancreatic-billiary diseases. They followed the proposition which divides these procedures in routine procedures, in evaluation and in development. In gallbladder diseases the laparoscopic cholecystectomy is considered gold-standard, taking advantage upon open cholecystectomy, excluding bile duct injury and cystic stump leakage. They emphasize the aspects related to acute cholecystitis, cholecystectomy during pregnancy, calcified gallbladder and cancer. The laparoscopic cholecystectomy during pregnancy is considered as a procedure in evaluation. Long term effects of pneumoperitonium on foetus are not well known. The approach in calcified gallbladder, because the high incidence of adenocarcinoma, remains questionable. Perspectives of videosurgery in complications of laparoscopic cholecystectomy are commented. Cystic stump leakage, misplaced clips and partial section or laceration of main biliary tract could be treated by new videosurgery in selected situations. In the treatment of choledocolithiasis, they consider the procedure as one for evaluation, due to the lack of randomized prospective trials. Until now, videocholedocolitotomy is reserved to specialized centers. In pancreatic diseases, videosurgery is a procedure in development, except for the non-complicated acute biliary pancreatitis, in which benefits with laparoscopic cholecystectomy has been shown. The same occurs with diversion surgeries for desobstruction of the biliary tree. Although performed within the conventional surgery principles there is a lack of comparative studies with other techniques analysing efficacy and efficiency.
\end{abstract}

Key Words: Videolaparoscopic cholecystectomy; Videosurgery; Videocholedocolithotomy; Biliary tract; Pancreas.

\section{REFERÊNCIAS}

1. Mühe E. Die erste cholezystektomie durch das laparoskop. Langenbecks Archiv Klin Chir 1986; 369:804.

2. Dubois F, Berthelot G, Levar H. Laparoscopic cholecystectomy. Historic perspective and personal experience. Surg Laparosc End 1991; 1:52-57.

3. IH Consensus Conference. Gallstones and Laparoscopic Cholecystectomy. JAMA 1993; 249:1.018-1.024.

4. Alves AS, Silva AL, Oliveira CA et al. Colecistectomia convencional, por minilaparotomia e videolaparoscopia. Rev Col Bras Cir 1997; 24:143-146.

5. de Pouvourville G; Ribet-Reinhart N; Fendrick M et al. A prospective comparison of costs and morbidity of laparoscopic versus open cholecystectomy. Hepatogastroenterology 1997; 44:35-9.

6. Deziel DJ, Millikan KW, Economou SG et al. Complications of laparoscopic cholecystectomy: a national survey of 4,292 hospitals an analysis of 77,604 cases. Am J Surg 1993; 165:9-14.

7. McMahon AJ, Fullarton G, Baxter JN et al. Bile duct injury and bile leakage in laparoscopic cholecystectomy. Br J Surg 1995; 82:307-313.

8. Doctor N, Dooley JS, Dick R et al. Multidisciplinary approach to biliary complications of laparoscopic cholecystectomy. Br J Surg 1998; 85:627-632.

9. Clavien PA, Sanabria JR, Mentha G et al. Recent results of elective open cholecystectomy in a North American and European Center. Comparision of complications and risk factors. Ann Surg 1992; 216:618-26.
10. Morgenstern BM, Wong L, Berci G. Twelve hundred open cholecystectomies before the laparoscopic era. A standard for comparision. Arch Surg 1992; 127: 400-3.

11. Wolfe BM, Gardiner BM, Leary BF et al. Endoscopic cholecystectomy. An analysis of complications. Arch Surg 1991; 126:1.192-8.

12. Raute M, Podlech P, Jaschke W et al. Management of bile duct injuries and strictures following cholecystectomy. World J Surg 1993; 17:553-562.

13. Rohde L, Borges da Costa MST, Wendt LR et al. Iatrogenic biliary strictures: surgical experiences with 39 patients. HPB Surg 1997; 10:221-227.

14. Park YH, Oskanian Z. Obstructive jaundice after laparoscopy cholecystectomy with electrocautery. Am Surg 1992; 58:321-3.

15. Herzog U, Messmer P, Sutter M et al. Surgical treatment for cholelithiasis. Surg Gynecol Obstet 1992; 175:238242.

16. Pickleman J, Gonzalez RP. The improving results of cholecystectomy. Arch Surg 1986; 121:930-934.

17. Peck JJ. Endoscopic cholecystectomy. An analysis of complications (comments). Arch Surg 1991; 126:3-12.

18. Rohde L, Borges da Costa MS, Osvaldt AB et al. Biliary complications of laparoscopic cholecystectomy. Congress of the International Hepato-Pancreato-Biliary Association, Budapest, 1999; 1:67-71.

19. Michalowski K, Bormann PC, Krige JE et al. Laparoscopic subtotal cholecystectomy in patients with complicate acute cholecystitis or fibrosis. Br J Surg 1998; 904-6. 
20. Davis A, Katz VL, Cox R, et al. Gallbladder disease in pregnancy. J Reprod Med. 1995; 40:759-62.

21. Galan HL, Reedy MB, Bean JD et al. Maternal and fetal effects of laparoscopic insufflation. Anesthesiology 1994; 81:31-36.

22. Hunter J, Swanstrom L, Thornburgh K. Carbon dioxide pneumoperitoneum induces fetal acidosis in a pregnant ewe model. Surg Endosc. 1995;9:272-279.

23. Elerding S C. Laparoscopic cholecystectomy in pregnancy. Am J Surg 1993; 165:625-627.

24. Polk HC. Carcinoma and calcified gallbladder. Gastroenterology 1966; 50:582-5.

25. Razzetta F, Borgonovo G, Cagnazzo A et al. Laparoscopic cholecystectomy and gallbladder cancer: a diagnostic and therapeutic dilemma. Eur J Surg Oncol 1997; 23:8485.

26. Z'graggen K, Birrer S, Maurer CA et al.Incidence of port site recurrence after laparoscopic cholecystectomy for preoperatively unsuspected gallbladder carcinoma. Surgery 1998; 124:831-8.

27. Kolecki R, Schirmer B. Intraoperative and laparoscopic ultrasound. Surg Clin North Am 1998; 78:251-271).

28. Kim H, Roy T. Unexpected Gallbladder Cancer with cutaneous seeding after laparoscopic cholecystectomy. South Med J 1994; 87:817-820.

29. Zeliotto Junior A, Mendes JAM, Künzle JE et al. Metástases de adenocarcinoma de vesícula biliar em colecistectomia videolapararoscópica. Rev Col Bras Cir 1998; 25:357-8.

30. Perim CA, Guedes, MAE. Colecistectomia laparoscópica: experiência em 500 casos, enfatizando as vantagens da colecistocolangiografia e da ligadura do ducto e a artéria cística utilizando fio de náilon. Rev Col Bras Cir 1999; 26:39-43.

31. Petelin J. Laparoscopic approach to common duct pathology. Surg Laparosc Endosc 1991; 1:33-41.

32. Traverso LW, Haargeave K, Kazarek RA. A cost-effective approach to the treatment of common bile duct stones with surgical versus endoscopic techniques. In: Bile ducts and bile duct stones. Berci, Cushieri, WB Saunders Company, 1997, pp. 154-160.

33. De Paula AL, Hashiba K, Bafutto M. Laparoscopic management of choledocholitiasis. Surg Endosc 1994; 8:13991403.

34. Gurbuz AT, Watson D, Fenoglio ME. Laparoscopic choledochoduodenostomy. Am Surg 1999; 65:212-214.

35. Heili MJ, Wintz N K, Fowler DL. Choledocholitiasis: endoscopy versus laparoscopy management. Am Surg 1999, 656:135-8.

36. Ballestra-Lopez C, Batisda-Vila X, Bettonica-Larrañaga C et al. Laparoscopic management of acute biliary pancreatitis. Surg Endosc 1997; 11:718-21.
37. Targarona EM, Pera M, Martinez, Balague C et al. Laparoscopic treatement of pancreatic disorders: diagnosis and staging, palliation of cancer and treatment of pancreatic pseudocysts. Int Surg 1996; 81:1-5.

38. Warshaw $\mathrm{Al}, \mathrm{Gu} \mathrm{Z}$, Wittenberg $\mathrm{J}$ et al. Preoperative staging and assessment of ressecability of pancreatic cancer. Arch Surg 1990; 230-3.

39. Merchant NB, Conlon KC, Saigo P et al. Positive peritoneal cytology predicts unresectabillity of pancreatic adenocarcinoma. J Am Coll Surg 1999; 188:421-6.

40. Chapuis Y, Bigourdan JM, Massault PP et al. Videolaparoscopic excision of insulinoma. A study of 5 cases. Chirurg 1998; 123:461-7.

41. Matsumoto T, Kitano S, Yoshiba T et al. Laparoscopic resection of pancreatic mucinous cystadenoma using laparosonic coagulating shears. Surg Endosc 1999; 13:172-3.

42. Gagner M, Pomp A. Laparoscopic pylorus-preserving pancreaticoduodenectomy. Surg Endosc 1994; 8:408-10.

43. Schöb OM, Schmid RA, Morimoto AK et al. Laparoscopic Roux-en-Y choledochojejunostomy. Am J Surg 1997; $173: 312-9$

Endereço para correspondência

Prof. Luiz Rohde

Rua Coronel Bordini, 1535/501

90440-001 — Porto Alegre-RS

E-mail: rohdepoa@zaz.com.br 